\title{
Pulsed release of antidepressants from nanocomposite hydrogels
}

\author{
Mario Casolaro $^{1 *}$ and Ilaria Casolaro ${ }^{2}$ \\ ${ }^{1}$ Department of Biotechnology, Chemistry and Pharmacy, University of Siena, Via Aldo Moro 2, Siena 53100, Italy \\ ${ }^{2}$ Psychiatry Resident, University of Siena, Siena 53100, Italy
}

\begin{abstract}

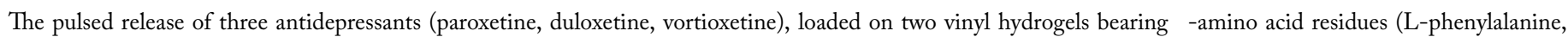
L-valine) and embedded magnetic nanoparticles, was evaluated in different buffer solutions of purposely chosen pHs (2.9, 4.6, and 7.4). At low pH values the release of the drug was always small for a long period of days, and it underwent a sudden increase when a solution of $\mathrm{pH} 7.4 \mathrm{was}$ maintained for a short period of hours. The releasing increase becomes much higher as greater was the residence time of the solution in contact with the hydrogel. The application of an alternating magnetic field $(\mathrm{AMF})$ further increases the amount of drug released during the $\mathrm{pH}$ pulses. These different release variations were related to a different and reversible ability to swell of the hydrogel. The mechanism behind the process was mainly due to the different acid/base behaviour of the free carboxyl groups.
\end{abstract}

\begin{abstract}
Abbreviations
AMF: Alternating magnetic field; EDS: Equilibrium degree of swelling; MDD: Major depressive disorder; SSRI: Selective serotonin reuptake inhibitors; EBA: $N, N^{\prime}$-ethylene-bisacrylamide; PHENip3: $\quad \operatorname{Poly}(N$-acryloyl-L-phenylalanine-co- $N$-isopropylacrylamide) hydrogel; AVA-5: Poly(N-acryloyl-L-valine) hydrogel crosslinked with $5 \mathrm{~mol} \%$ of EBA; AVA-2: Poly( $N$-acryloyl-L-valine) hydrogel crosslinked with $2 \mathrm{~mol} \%$ of EBA
\end{abstract}

\section{Introduction}

In recent years we have proposed some polyelectrolyte hydrogels, of anionic and ampholyte nature, capable of complexing, besides metal ions, with metal-based drugs and drug molecules containing amino functionality [1-3]. The main polymer structure bearing residues of $\triangle$-amino acids (L-phenylalanine, $\mathrm{L}$-valine, $\mathrm{L}$-histidine) was cross-linked with different cross-linking agents and copolymerized with $\mathrm{N}$-isopropylacrylamide (NIPAAm). In some cases, magnetic nanoparticles have been incorporated to make the hydrogel a multiresponsive nanocomposite sensitive to both direct $(\mathrm{pH}$, temperature, ionic strength) and remote (alternating magnetic fields, AMF) stimulations [3,4-6]. In the ionized state some of these hydrogels exhibit an exceptionally high degree of swelling, the magnitude of which can be monitored by the ionic strength, temperature and $\mathrm{pH}$. In fact, anionic hydrogels show different critical conditions at which the whole swollen mass completely collapse at $\mathrm{pH} 4$ and $\mathrm{pH} 5$ due to the compounds bearing the $\mathrm{L}$-valine and the L-phenylalanine residues, respectively [1-3]. Such critical $\mathrm{pH}$ values are related to their thermodynamic properties, as revealed from the study of the free polymers in aqueous solution [7-8]. A partial neutralization of the ionized carboxyl groups allows the shrinking of the macromolecular structure, as hydrophobic interactions outweight the electrostatic repulsion. Typically, the swollen hydrogel undergoes a rapid collapse in the presence of cationic drugs and this collapse is the more rapid and complete the larger is the basicity constant of the drug itself [2,9]. The main drug-polymer interaction was reported to be of ionic nature, although hydrophobic interactions cannot be excluded. It follows that the drug-polymer strength affects the whole drug release process; the free drug, that is in equilibrium with the adduct, must spread through the network to be used for therapeutic purposes. A further advantage is the load of the drug within the hydrogel, which preserves its effectiveness over time. This was evidenced by previous results for the 'in vitro' release study of anticancers (cis-platinum, doxorubicin) [10,11], glaucoma treatment (pilocarpine) [12] and, not finally, of antidepressants (citalopram, trazodone, paroxetine, duloxetine) [5,9]. In all cases, the typical release profiles showed that the drug concentration is maintained in the therapeutic window for an extended period of time, ensuring the sustained therapeutic action. In some cases, the need to promote a drug concentration in solution is ensured by the increasing temperature and by the application of the appropriate AMF stimulation; thus, 'on demand' may be released a greater amount of drug to the target organ to alleviate the medical needs of the moment and to restore a normal state of health $[2,4,5]$. Besides the sustained release formulation, the pulsatile system may benefit from a therapy mainly because it is linked with the circadian rhythms of the body $[13,14]$. Peppas et al. reported one of the first example of pulsatile local delivery of thrombolytic (streptokinase) and antithrombotic (heparin) drugs using temperature/pH responsive hydrogels based on NIPAAm copolymerized with methacrylic acid $[15,16]$. The hydrogels improved the pulsatile streptokinase release and the release rate between the swollen and collapsed hydrogel states was at least one order of magnitude different. Because of the many diseases that follow the circadian rhythms of the body, the goal in drug delivery

Correspondence to: Mario Casolaro, Department of Biotechnology, Chemistry and Pharmacy, University of Siena, Via Aldo Moro 2, Siena 53100, Italy, Tel.: +39-0577-234388; E-mail: mario.casolaro@unisi.it

Key words: stimuli-responsive hydrogels; pulsed release; paroxetine; duloxetine; vortioxetine

Received: October 15, 2017; Accepted: November 03, 2017; Published: November 06, 2017 
research is to develop formulations to meet therapeutic needs relating to particular pathological conditions. Researchers have addressed the question of creating new stimuli-responsive hydrogels as carriers for drug delivery systems because their unique physicochemical and biological properties can protect sensitive drugs $[17,18]$. Based on the externally triggered ability, the use of stimuli-responsive hydrogels can be associated with the events of the circadian rhythm that cause the release of a drug at predetermined intervals.

Following our interest in the release of antidepressants, this paper is concerning with the results of the pulsed release behaviour of three drugs (Scheme 1: paroxetine, duloxetine, vortioxetine) by nanocomposite hydrogels used previously.

Depression is a common illness worldwide and can happen to anybody; it causes mental anguish and affects people's ability to carry out everyday tasks [19]. Recently, the World Health Organization has estimated more than 300 million people to be affected and, for this purpose, has launched a one-year campaign to ensure that more people with depression both seek and get help [20]. Fortunately, depression can be prevented and treated adequately. For the treatment of the disease, the three drugs mentioned above are specific and used today in the clinical practice. Like the paroxetine, both duloxetine and vortioxetine are two new antidepressant drugs that have been used clinically in the treatment of major depressive disorder (MDD) [21]. A variety of meta analyses have been conducted to evaluate the efficacy and tolerability of these three drugs in depression treatment [22]. It was concluded that, whereas the paroxetine is superior or equivalent to placebo and that it is equivalent or inferior to other antidepressants, duloxetine was more effective but less well-tolerated than vortioxetine [23]. Antidepressants, of a basic nature for the presence of the secondary amino nitrogen atom, were loaded on two anionic polyelectrolyte hydrogels bearing L-phenylalanine (PHE-Nip3) and/or L-valine (AVa-5) residues [9]. While the first platform is formed by a copolymer (poly- $N$-acryloylL-phenylalanine-co-NIPAAm in a molar ratio of one and crosslinked with $N, N^{\prime}$-ethylene-bis-acrylamide, EBA, of $2 \mathrm{~mol} \%$ ), the second one consists of a homopolymer (poly- $N$-acryloyl-L-valine) crosslinked with EBA of $5 \mathrm{~mol} \%$. In addition, the hydrogel PHE-Nip3 contains embedded magnetic nanoparticles $\left(\mathrm{CoFe}_{2} \mathrm{O}_{4}\right)[4]$.

With the same drug, the different thermodynamic properties of the polymer enable the design of drug carrier systems that are best suited to the application purpose. The release of antidepressants was conducted in aqueous solution of acid $\mathrm{pH}$ for a long period of time with a short overlap period of regular pulses at basic $\mathrm{pH}$. In this short time interval, the hydrogel is subjected to the stimulus of a high $\mathrm{pH}$ value that involves two simultaneous processes: a) an increase in swellability and b) a chemical equilibrium displacement towards the dissociation of the drug bound to the polymer. The return to low acidic $\mathrm{pH}$ conditions restores the low swelling/collapse of the network with a minor drug loading. In this work it is reported the pulsed release behaviour of some antidepressant drugs, of interest as a template for the design of all the transport systems of drugs for the therapy of diseases which follow the circadian rhythms of the body [24].

\section{Results and discussion}

\section{Pulsed release of paroxetine}

Paroxetine is an antidepressant belonging to the class of Selective Serotonin Reuptake Inhibitors (SSRI) [25]. Due to its efficacy and good tolerability, it is commonly used in the treatment of MDD [21]. In particular, for its marked sedative effect, it has successfully been demonstrated to improve mood disorders with anxiety characteristics and/or even anxiety disorders such as panic disorder, generalised anxiety and social fobia, at a dosage ranging from 20 to $60 \mathrm{mg} /$ day. The main side effects are attributable to an anticholinergic feature responsible for dry mouth, constipation, sedation; for the same reason it is controindicated in case of glaucoma and benignal prostatic hypertrophy. Nausea could occur in the first weeks of treatment with paroxetine and it could be prevented by a full stomach administration. Sedation often requires an evening administration. No risk for addiction or tolerance occur but, due to its short half-life, discontinuation symptoms (such as nausea, headache, dizziness, sleep problems, anxiety) could appear if a slow dosage reduction is not provided. Thus, a long-lasting sustained release of the drug could go beyond this limit. The pulsed release of the paroxetine from hydrogels PHE-Nip3 and AVA-5 was analyzed in a period of time long enough (at least two weeks) in two acidic $\mathrm{pH}$ solutions ( $\mathrm{pH} 2.9$ and 4.6). At set intervals of time, the external acid solution was replaced by a physiological $\mathrm{pH}$ solution (PBS, $\mathrm{pH}$ 7.40) for a short period of time ( 1 or 2 hours). The choice of these $\mathrm{pH}$ values was dictated by the swelling behaviour of the hydrogels, as previously reported $[2,9]$. While the thermodynamic properties of the hydrogel PHE-Nip3 showed a critical point of collapse at $\mathrm{pH}$, the hydrogel AVA-5 involves a collapse at $\mathrm{pH} 4$. The basic difference is to be attributed to the chemical structure of the two polymers. In fact, while the completely ionized and swollen hydrogel carrying the L-phenylalanine residues show a shrinking phenomenon at a degree of protonation $\otimes=0.3$, the hydrogel with the L-valine residues shrinks to $\bigotimes$ value of about twice [9]. The different behaviour was attributed to the greater hydrophobic attraction effect which clearly exceeds the ion repulsive effect exerted by the remaining $\mathrm{COO}^{-}$groups, and this, for the hydrogel PHE-Nip3, is early compared to the hydrogel AVA-5. So, for the two hydrogels it is to expect a more swollen state at $\mathrm{pH} 7.4$, and a state of collapse at $\mathrm{pH} 2.9$; on the other hand, at $\mathrm{pH} 4.6$ a collapsed state should only occur for the hydrogel PHE-Nip3. Moreover, the presence of the ionized drug substantially contributes to maintain the hydrogel in the collapsed state because of its ionic interaction with the carboxylic groups. This is evident in any case since the formation of the drug-hydrogel adduct leads to the complete collapse of the ionic and swollen hydrogel in presence of the hydrochloride drug solution $[4,5,9]$. Additionally, despite the non-swellability at acid $\mathrm{pH}$, the hydrogel loaded with the drug is wettable and capable of releasing the drug itself in a slow way and controllable by external stimuli.

In Figure 1 is shown the comparison of the pulsed release of the paroxetine from the hydrogel PHE-Nip3 in two different aqueous solutions of $\mathrm{pH} 2.9$ and 4.6 , with the $\mathrm{pH}$ pulse 7.4 for 1 hour. In addition to $\mathrm{pH} 4.6$, it is shown a comparison with the pulse of $\mathrm{pH} 7.4$ for 2 hours. In all cases, the additional stimulation of the alternating magnetic field (AMF) is compared.

From the beginning it is immediately evident that the release of the drug is greater in the more acidic solution $(\mathrm{pH} 2.9)$ and that the released quantity becomes significantly higher when an appropriate external AMF of low frequency $(20 \mathrm{kHz}, 50 \mathrm{~W})$ is applied. In general, the application of the AMF produces an increase in the percentage of release, especially at the first stage of the pulsed release [2,9]. This because the energy induced by the AMF can cause an improved permeability control mechanism due to oscillation or vibration of the network-embedded $\mathrm{CoFe}_{2} \mathrm{O}_{4}$ magnetic nanoparticles $[4,6]$. This in turn may cause twisting and/or displacement of the polymeric chains, resulting in an enhancement of the diffusion process. According to other researchers, the magnetically-induced deformation of the hydrogel is a result of the elastic oscillation or vibration of the embedded magnetic 
<smiles>Fc1ccc([C@H]2CCNC[C@H]2COc2ccc3c(c2)OCO3)cc1</smiles>

Paroxetine<smiles>CNCC[C@H](Oc1cccc2ccccc12)c1cccs1</smiles>

Duloxetine<smiles>Cc1ccc(Sc2ccccc2N2CCNCC2)c(C)c1</smiles>

Vortioxetine

Scheme 1. Chemical structure of antidepressant drugs.
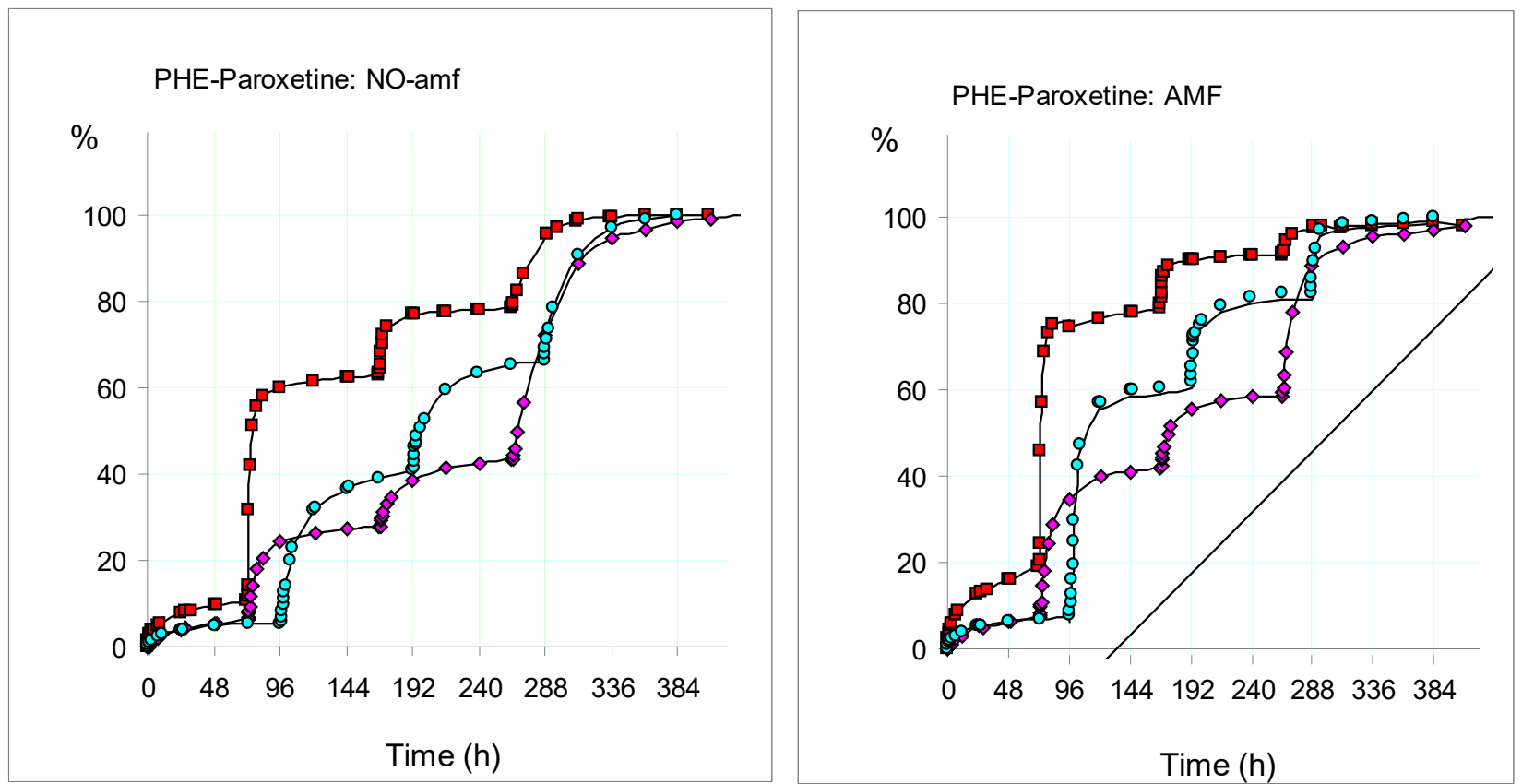

Figure 1. Release of paroxetine (\%) from the hydrogel PHE-Nip3 in buffered solutions (pH 4.60: pink rhomboidal and light blue circles; pH 2.9: red squares) with PBS (pH 7.40) pulsed variations of $1 \mathrm{~h}$ (pink, red) and $2 \mathrm{~h}$ (light blue) without (left panel) and with (right panel) AMF applications $(20 \mathrm{kHz} / 50 \mathrm{~W})$ at $25^{\circ} \mathrm{C}$. The results are the average of at least two experiments with less than $3 \%$ error.

nanoparticles resulting in mechanical deformation of the hydrogel to squeeze out the drug (26-28). On the other hand, the release into solution at $\mathrm{pH} 4.6$ is significantly lower for a long period of time. Only the change of external solution to $\mathrm{pH} 7.4$ (first $\mathrm{pH}$ pulse of 1 hour) leads to a significant increase in the paroxetine release which is even greater if the residence time in contact with the hydrogel is doubled to two hours: compared with 1-hour, pulses of 2-hours ensure a higher percentage of the drug in solution. The return to conditions of acidic $\mathrm{pH}$ (2.9 and 4.6) restores the release for the successive period of days, while the further pulse to $\mathrm{pH} 7.4$ reproduces the burst in drug release. Finally, in the last $\mathrm{pH}$ pulse performed at stage 3, the PBS solution at $\mathrm{pH} 7.4$ has been in contact for a long period of days to allow the complete release of the drug from the hydrogel. This sustained release has required a few days, despite the speeding of the AMF force [9].

Of course, all the stepwise process is to be related to chemical equilibria of all ionic/ionizable species involved and their base strength. Paroxetine, being a strong base $(\operatorname{logK} 9.77)[9,29]$, remains mostly in the protonated state in all considered $\mathrm{pH}$ conditions. This species, interacting ionically to some extent with the ionized $\mathrm{COO}^{-}$groups of the swollen hydrogel, allows the collapse of the whole structure with the formation of a drug-polymer adduct of chemical stability. Possible $\mathrm{pH}$ variation of a solution containing the adduct may shift the protonation equilibrium of the hydrogel towards the ionised form if the $\mathrm{pH}>\mathrm{pKa}$ (pKa of the hydrogel) [2,9] with the consequence of an increase in swellability and a greater release of the drug in solution; on the other hand, at $\mathrm{pH}<\mathrm{pKa}$ the hydrogel involves a charge neutralization with a consequent shrinking phenomenon and a greater availability of the free drug in solution. All this is consistent with the data reported in Figure 1 .

We can find a support mechanism in the paroxetine release data from the hydrogel AVA-5 in the same $\mathrm{pH}$ conditions used for the hydrogel PHE-Nip3. As reported in Figure 2, once again the release of the drug at $\mathrm{pH} 2.9$ was slightly higher than that experienced at $\mathrm{pH} 4.6$, despite the greater swellability of the hydrogel AVA-5 at this latter $\mathrm{pH}[3,5,9,12]$. 


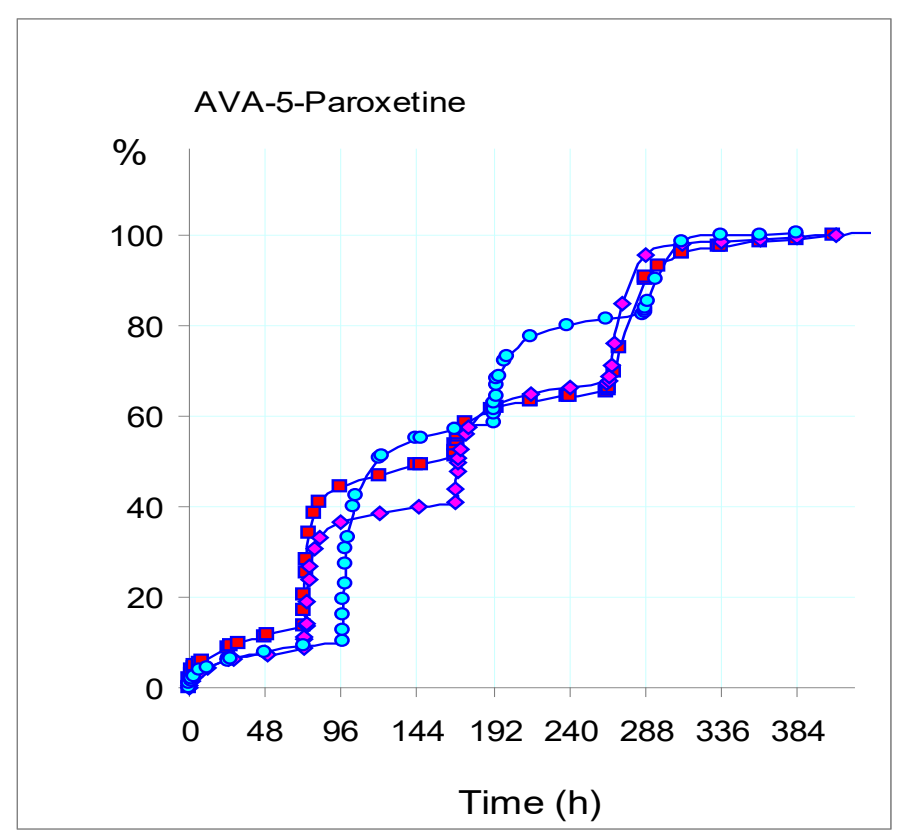

Figure 2. Release of paroxetine (\%) from the hydrogel AVA-5 in buffered solution ( $\mathrm{pH}$ 4.60: pink rhomboidal and light blue circles; $\mathrm{pH}$ 2.9: red squares) with PBS ( $\mathrm{pH} 7.40$ ) pulsed variations of $1 \mathrm{~h}$ (pink, red) and $2 \mathrm{~h}$ (light blue) at $25^{\circ} \mathrm{C}$. The results are the average of at least two experiments with less than $3 \%$ error.

In fact, the greater swellability involves a burst release of paroxetine, as observed at the $\mathrm{pH}$-pulse of 7.40; this does significantly increase the released amount for both $1 \mathrm{~h}$ and $2 \mathrm{~h}$ pulses. It is worth-noting that the greater cross-linked hydrogel AVA-5 releases a quantity of paroxetine comparable to that shown by the hydrogel PHE-Nip3 in the same conditions of $\mathrm{pH}$ and even under stimulation of the AMF. A minor release is only observed at $\mathrm{pH} 2.9$ due to the compactness of the adduct and the greater value of $\mathrm{pKa}$ for the hydrogel AVA-5 [2,9].

At the end of each pulsed release process, the hydrogel showed a fairly constant swelling with values of equilibrium degree of swelling (EDS) between 30 and 35 for the hydrogel PHE-Nip3 and between 15 and 20 for the hydrogel AVA-5. The value of the equilibrium degree of swelling (equation 1)

$$
\mathrm{EDS}=\left(\mathrm{W}_{\mathrm{w}}-\mathrm{W}_{\mathrm{d}}\right) / \mathrm{W}_{\mathrm{d}}
$$

(where $\mathrm{W}_{\mathrm{w}}$ and $\mathrm{W}_{\mathrm{d}}$ is the weight of wet and dry hydrogel, respectively) represents the state of the wettability of the hydrogel, i.e. the quantity of bound water per unit of dry weight of the hydrogel itself. Of course this depends on the $\mathrm{pH}$ value, as the gradual ionization of the hydrogel promotes its swelling. In all the intermediate stages, the EDS follows a trend connected to the $\mathrm{pH}$ and to the amount of the drug released at that stage. Figure 3 summarizes the results of the EDS values obtained for the release of paroxetine from the hydrogel PHENip3 at the three different $\mathrm{pH}$ values considered and with time-pulse of $1 \mathrm{~h}$ and $2 \mathrm{~h}$. The same figure 3 shows for comparison the behaviour of the hydrogel AVA-5 in the same $\mathrm{pH}$ conditions.

At a first look it appears a reversible swelling-collapsing process for the hydrogel PHE-Nip3 when the $\mathrm{pH}$ varies from 2.9 (or 4.6) to 7.4; a low value of EDS at acid $\mathrm{pH}$ is compatible with the complete charge neutralization of the network. The gradual release of the drug in the successive pulses at $\mathrm{pH} 7.4$ improves the formation of free $\mathrm{COO}^{-}$groups that consequently leads to an increase in the hydrogel swellability. Though small, some significant changes in EDS can be observed in the AMF regime for $\mathrm{pH}$ pulses of $1 \mathrm{~h}$ or $2 \mathrm{~h}$. The small increments of the EDS values are in line with the increased release of the drug that results in greater ionization of the hydrogel, especially at $\mathrm{pH}$ 7.40. On the other hand, the hydrogel AVA-5, despite its greater cross-linking content, shows a clear trend of EDS which is confirming the hypothesis of a greater swelling for an increased ionisation, due to the release of the drug. Besides the clear reversibility observed for the swollen-collapse at $\mathrm{pH} 2.9$, with pulse of $1 \mathrm{~h}$ at $\mathrm{pH} 7.4$, no collapse at $\mathrm{pH} 4.6$ is observed, but rather there is an increased swellability in the subsequent pulses, with values of EDS at $\mathrm{pH} 7.4$ always higher than those observed at $\mathrm{pH}$ 4.6. This trend is enhanced with a $2 \mathrm{~h}$ pulse. Of course, this different behaviour of the two hydrogels is to be related to thermodynamic properties described above [2,9].

\section{Pulsed release of duloxetine and vortioxetine}

Duloxetine is an antidepressant drug belonging to the class of Serotonin-Norepinephrin Reuptake Inhibitors [30]. It is commonly used in the treatment of depression, mainly in case of anhedonia and lack of energy. Even if it is well tolerated, sometimes symptoms of anxiety and hyperactivation could occur, along with gastrointestinal symptoms, dizziness and sleep disorders. The usual therapeutic dosage ranges from 30 to $120 \mathrm{mg}$; particular attention could be paid in case of older patients, who would benefit from a lower dosage. Duloxetine has been approved also for the treatment of neuropathic pain, even with a lot of caution due to the high risk for switching in maniac episodes in people suffering from undiagnosed Bipolar Disorder [31].

Vortioxetine is a recent antidepressant molecule having a multifactorial mechanism of action [32,33]. It shows an action on serotonin receptors, as well as on other neurobiological targets such as norepinephrine, dopamine, acetylcholine and histamine neurotransmissions. This multimodal feature provides a therapy for mood and anxiety disorders, but it even improves cognitive function, allowing to reach the "Full Functional Recovery" in depressed patients and also demonstrating to be of interest in the treatment of elderly mood disorders. The usual dosage ranges from 5 to $20 \mathrm{mg}$; the low incidence of side effects (mainly nausea) makes this new molecule suitable for safe long-term treatment of MDD. Thanks to its long halflife (66 hours), there is no risk for discontinuation symptoms.

In a similar manner to the release of paroxetine, the sustained release of duloxetine and vortioxetine by the hydrogel PHE-Nip3 was evaluated only at $\mathrm{pH} 4.60$ with pulse of $2 \mathrm{~h}$ at $\mathrm{pH} 7.40$ and under similar magnetic stimulations $(20 \mathrm{kHz}$ and $50 \mathrm{~W})$. Figure 4 summarizes the results obtained and compared with those of paroxetine reported above.

Since the initial stage, at $\mathrm{pH} 4.60$ it may see a release (\%) of duloxetine and vortioxetine appreciably higher than that of paroxetine, and this is further improved in the AMF regime. With the application of the first pulse at $\mathrm{pH} 7.40$, the burst release of duloxetine and vortioxetine clearly exceeds that observed for paroxetine; these, though in larger quantity, are leveled to the same amount shown in the AMF regime. From the second stage of pulse at $\mathrm{pH} 7.40$, the release of vortioxetine is improved with respect to duloxetine, even if the effect of the AMF seems to be negligible in comparison to the superiority shown for the release of the paroxetine. In these release mechanisms, the strength of polymerdrug interaction plays a key role; as we have reported the interaction is of electrostatic type, and is basically related to the pKa of the drug [29]. At the same base strength of the polymer, a high pKa value of the drug produces a stronger electrostatic interaction and therefore a lower tendency to release the same drug in solution in equilibrium with the drug-polymer adduct. The system under study shows that 


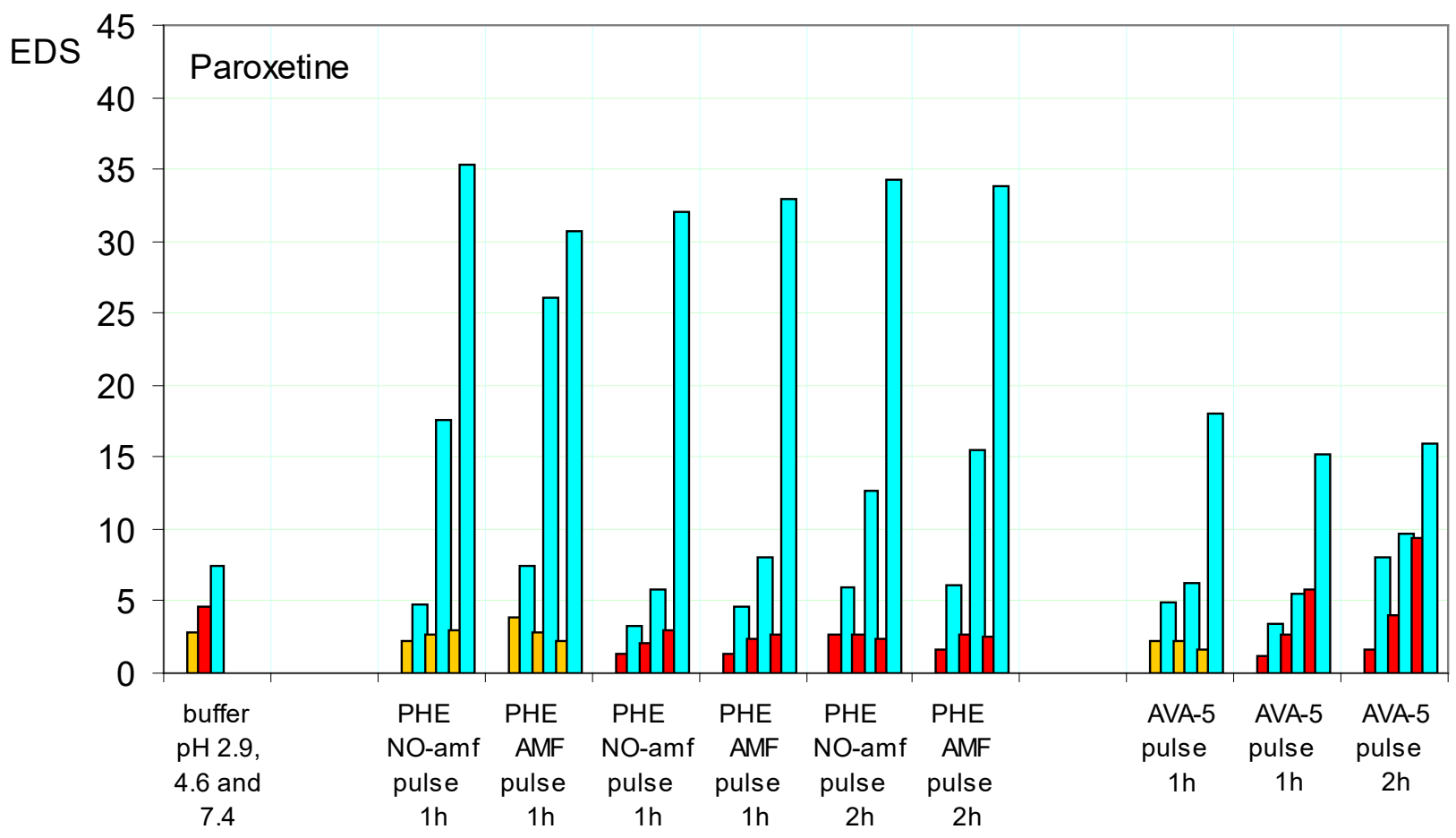

Figure 3. Equilibrium degree of swelling (EDS) of hydrogels PHE-Nip3 and AVA-5 after releasing paroxetine at pulsed variations (1h and $2 \mathrm{~h}$ ) of three buffer solutions at different $\mathrm{pH}$ values (2.9: orange; 4.6: red; 7.4: light blue) with and without AMF applications. The results are the average of at least two experiments with less than $8 \%$ error.
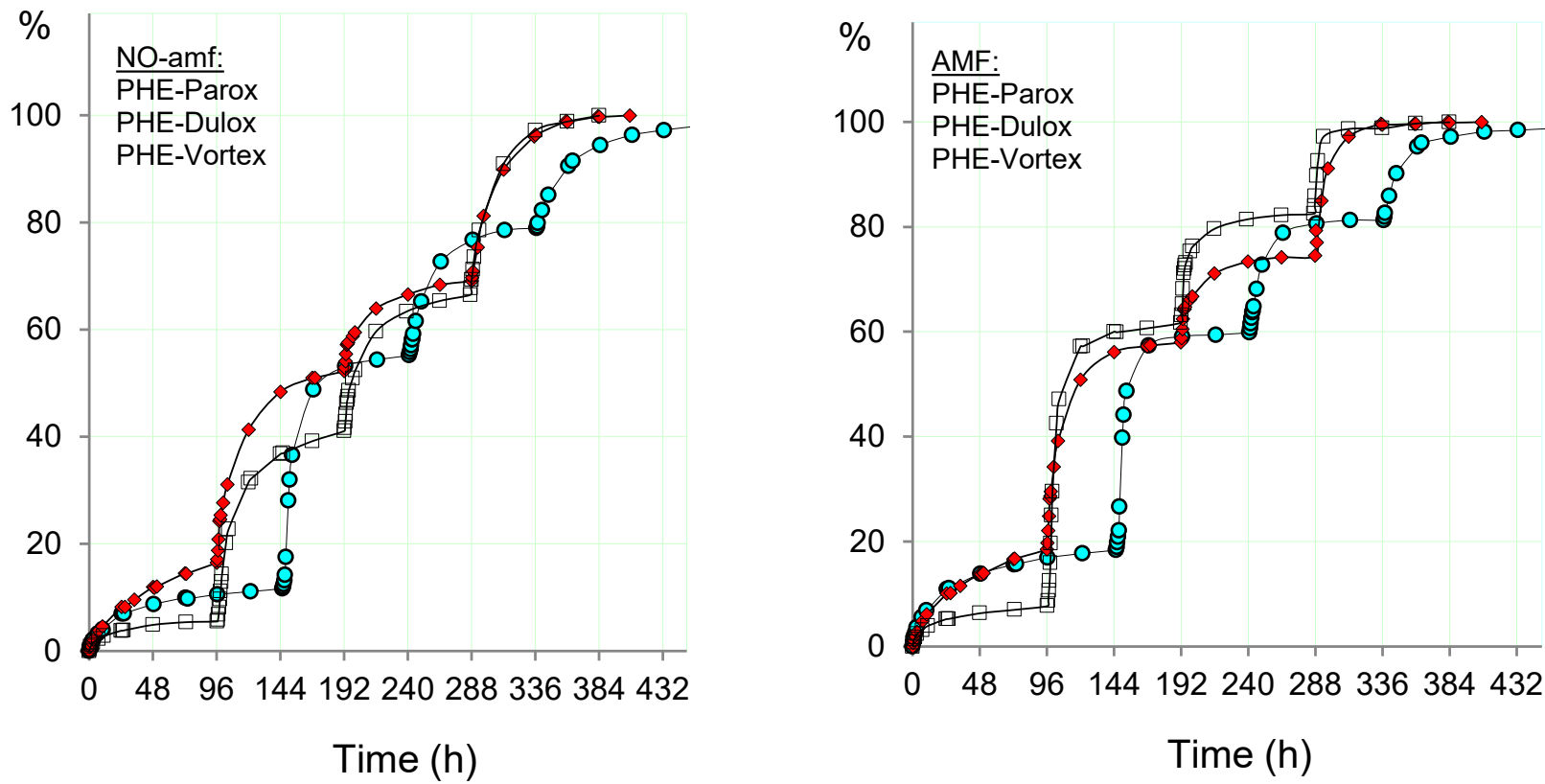

Figure 4. Release of antidepressant drugs (\%: paroxetine, squares; duloxetine, red rhomboidal; vortioxetine, light blue circles) from the hydrogel PHE-Nip3 in buffered solutions (pH 4.60) with PBS ( $\mathrm{pH} 7.40)$ pulsed variations of $2 \mathrm{~h}$ without (left panel) and with (right panel) AMF applications $(20 \mathrm{kHz} / 50 \mathrm{~W})$ at $25^{\circ} \mathrm{C}$. The results are the average of at least two experiments with less than $3 \%$ error.

the ionized paroxetine has a greater capacity for ionic interaction with the $\mathrm{COO}^{-}$groups of the polymer as its basicity (pKa 9.77) is higher than that of the other two drugs (duloxetine, pKa 9.7; vortioxetine, pKa 8.85) [29]. Also, the hydrophobic effects and solubility of these drugs play a significant role in the release process, especially in pulsed processes involving changes in $\mathrm{pH}$ and swellability of the polymeric network. This trend is supported by the release data of the same drugs from hydrogel with weaker base strength (AVA-5). In fact, Figure 5 reproduces, already from the first pulsed step, a release of paroxetine and duloxetine significantly higher than that observed for the hydrogel PHE-Nip3 under the same conditions.

Moreover, the greater cross-linking of the AVA-5 hydrogel drastically reduces the burst release of the duloxetine at $\mathrm{pH} 4.60$ that 


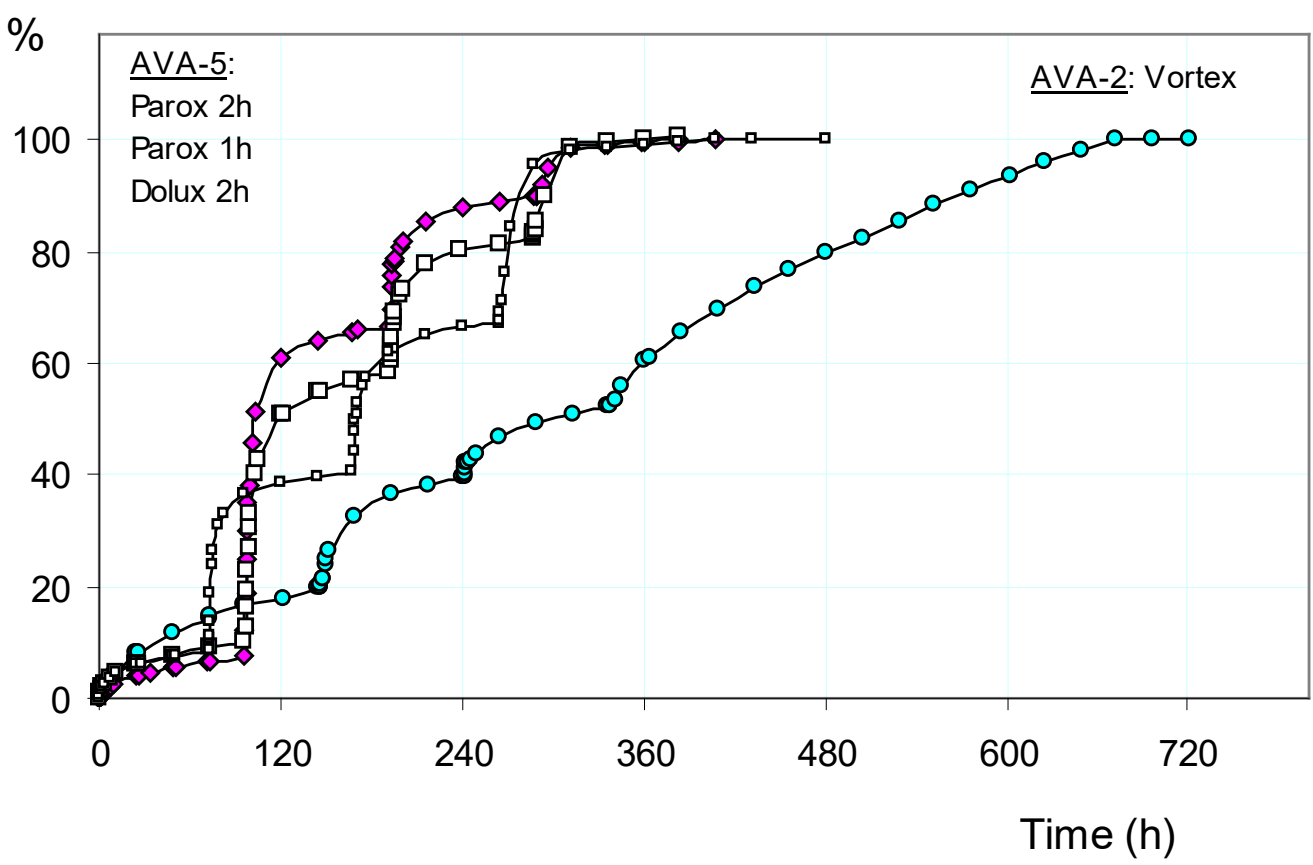

Figure 5. Release (\%) of: paroxetine (squares) and duloxetine (pink rhomboidal) from the hydrogel AVA-5; vortioxetine (light blue circles) from the hydrogel AVA-2 at $25^{\circ} \mathrm{C}$ and in buffered solutions ( $\mathrm{pH}$ 4.60) with PBS ( $\mathrm{pH} 7.40$ ) pulsed variations of $2 \mathrm{~h}$ (comparison with $1 \mathrm{~h}$ pulses is enclosed for paroxetine). The results are the average of at least two experiments with less than $3 \%$ error.

practically becomes similar to paroxetine. On the other hand, the hydrogel with the lowest degree of cross-linking (AVA-2) $[2,12]$ shows an initial release profile of vortioxetine which is higher than and in line with the general trend observed in all cases. The lower degree of cross-linking for the hydrogel AVA-2, resulting in greater swellability and comparable to the two values of $\mathrm{pH} 4.60$ and $\mathrm{pH}$ pulsed 7.40 , produces a release profile of vortioxetine significantly different from the other reported cases (Figure 5). This release becomes significantly more sustained for long periods and with less pulse effect. The degree of swelling (EDS) observed grows regularly with the successive pulses both at $\mathrm{pH} 4.60$ and $\mathrm{pH} 7.40$ because the hydrogel has never collapsed in any case; once again, the collapsing phenomenon occurs only for the hydrogel PHE-Nip3 that releases the drug, and at the various $\mathrm{pH}$ pulses it swells and collapses for the thermodynamic characteristics described above (Figure 6).

\section{Conclusions}

New stimuli-responsive hydrogels based on $\bigotimes$-amino acid residues can be considered as promising platforms for chrono-therapeutic applications, as they respond to external triggers [1,2,4,5,9-11]. The complications of circadian rhythms generally require a chronopharmacotherapy which can be easily accomplished by means of a suitable pulsed drug delivery system [13-18]. The pulsed release of drugs is a new delivery system that certainly provides an increase in therapeutic benefit to patients suffering from chronic diseases, because it delivers the drug in the right amount at the right time and place. Though the possible biomedical applications are remarkable, in this study we wanted to preliminarily investigate the pulsed release of antidepressant drugs from two types of hydrogels bearing residues of L-phenylalanine (PHE-Nip3) and L-valine (AVA-5 and AVA-2 ). Unlike the simple hydrogel of AVA series which however is basically sensitive to variations in $\mathrm{pH}$ and temperature, the PHE-Nip3 hydrogel contains embedded magnetic nanoparticles that make it sensitive to further stimulation of AMF. In fact, at two different acidic $\mathrm{pH}$ values $(\mathrm{pH} 2.9$ and 4.6) the pulsed release at physiological $\mathrm{pH}(\mathrm{pH}$ 7.4) of paroxetine is significantly higher in the presence of a lowfrequency AMF. The gradual and pulsed variation of the $\mathrm{pH}$ implies a swelling-collapsing mechanism of the hydrogel, which improves a higher release rate of the drug in dependence also on the time of the applied pulse. The pulsed release of duloxetine and vortioxetine show a closer paroxetine behaviour even if some observable differences are attributable to the lower basic strength and greater hydrophobicity of the drugs. These latter show a more weakly electrostatic interactions with the hydrogel and, consequently, are released with a higher rate. However, the mechanism by diffusion is insured in all cases when the swollen hydrogel to the pulse of $\mathrm{pH} 7.4$ is then collapsed at a $\mathrm{pH}$ lower than critical. On the basis of these preliminary observations we conclude that the proposed polyelectrolytes hydrogel platforms can be used to design useful chrono-pharmacotherapeutic systems based on the circadian rhythm of the body [13-16,24]. Among these systems, one that may deserves a certain interest is the design of an artificial pancreas. To this purpose, a study for the release of insulin from smart hydrogels containing the immobilized enzyme glucose oxidase was undertaken for future outlook [34].

\section{Experimental section}

\section{Materials and methods}

The three antidepressant drugs used in this study were obtained by purification of commercial tablets in methanol solution. Similarly to paroxetine and duloxetine reported previously [9], the vortioxetine was extracted by the tablets of Brintellix $10 \mathrm{mg}$ (H.Lundbeck A/5) in methanolic solution for $24 \mathrm{~h}$ under magnetic stirring. Subsequently, the solution was decanted and filtered over cotton. The dry product provided a fine white powder with consistent performance. Similarly, the three hydrogels used (PHE-Nip3, AVA-5 and AVA-2) have been reported previously with the loading of paroxetine and duloxetine by some of them $[2,4,9,12]$. Briefly, the hydrogel PHE-Nip3 consists of a 


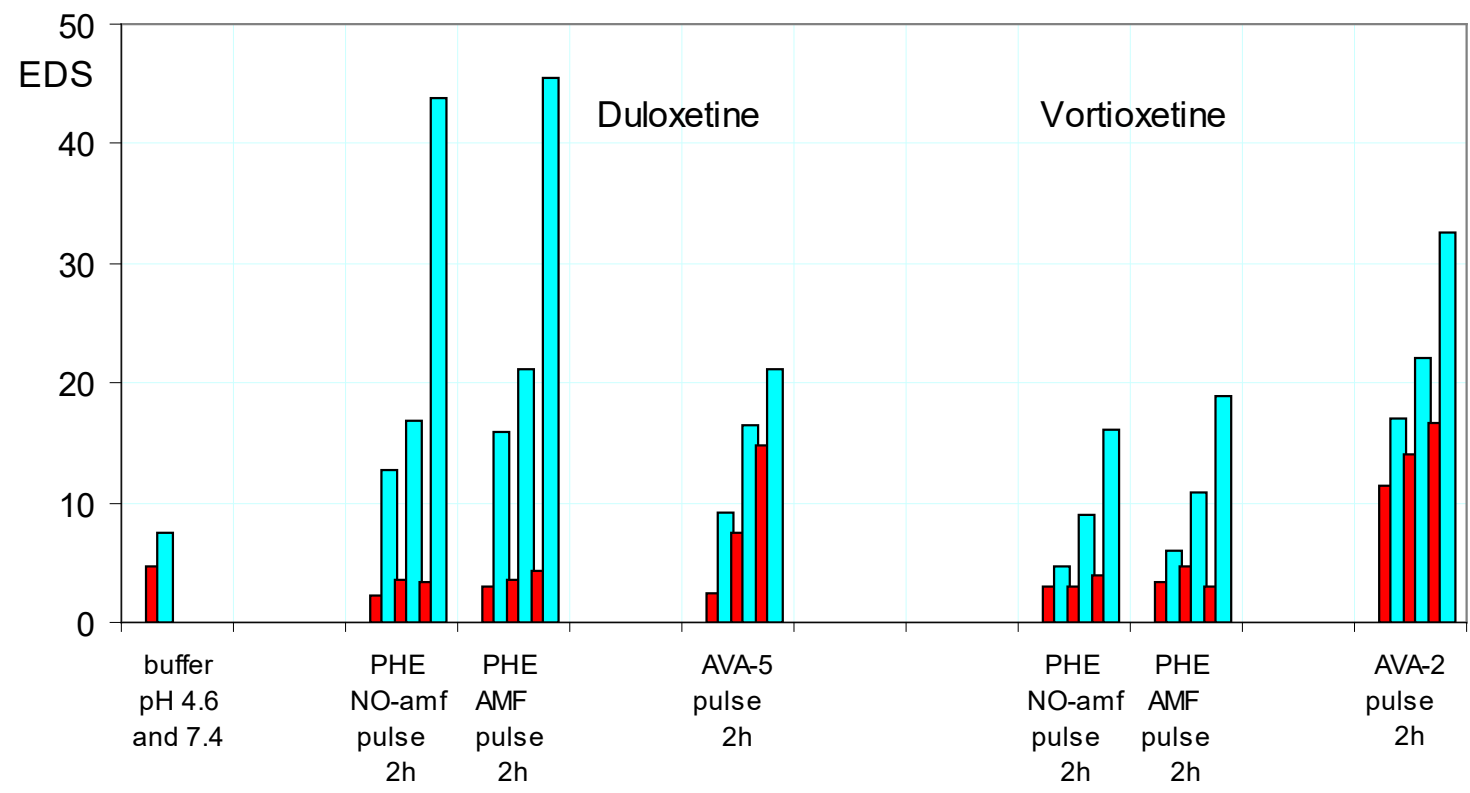

Figure 6. Equilibrium degree of swelling (EDS) of hydrogels PHE-Nip3, AVA-5 and AVA-2 after releasing duloxetine and vortioxetine at pulsed variations ( 2 h) of two buffer solutions at different $\mathrm{pH}$ values (4.6: red; 7.4: light blue) with and without AMF applications. The results are the average of at least two experiments with less than $8 \%$ error.

copolymer of $\mathrm{N}$-acryloyl-L-phenylalanine and $\mathrm{N}$-isopropylacrylamide units (in a molar ratio 1 to 1 ) and cross-linked with 2 mol\% of $N, N$ 'ethylene-bisacrylamide (EBA ); the cross-linking reaction was carried out in aqueous solution in the presence of cobalt ferrite $\mathrm{CoFe}_{2} \mathrm{O}_{4}$ magnetic nanoparticles [6]. On the other hand, the two homopolymer hydrogels were obtained by radical reaction of $N$-acryloyl-L-valine in the presence of 2 mol\% (AVA-2) and 5 mol\% (AVA-5) of cross-linking agent EBA. The three buffer solutions were prepared at three different $\mathrm{pHs}$ and at ionic strength $0.15 \mathrm{~mol} / \mathrm{L} \mathrm{NaCl}$. While the $\mathrm{PBS}$ solution at $\mathrm{pH}$ 7.40 and the acetate solution at $\mathrm{pH} 4.60$ were prepared according to the recipe shown in a previous work [9], the solution at $\mathrm{pH} 2.90$ was made up of hydrochloric acid added to the saline solution by controlling the $\mathrm{pH}$ thoroughly with a $\mathrm{pH}$-meter. The $\mathrm{pH}$ measurements were carried out with a potentiometric apparatus (TitraLab90, Radiometer Analytical) and automatically recorded with a Windows-based software (TimTalk 9). A combined glass $\mathrm{pH}$ electrode (Red Rod) and a temperature sensor (T201) carefully measured the $\mathrm{pH}$ and the temperature of the solution [35]. Before each $\mathrm{pH}$ measurement, the apparatus was calibrated with two buffer solutions (Radiometer Analytical) at $\mathrm{pH} 4.01$ and $\mathrm{pH}$ 7.00. The spectrophotometric measurements were made with a Specord 210 spectrophotometer (Analytik Jena) equipped with 10 $\mathrm{mm}$ quartz cuvettes. In addition, the alternating magnetic field (AMF) measurements have been supported by an AG 1006 amplifier/generator (T\&C Power Conversion, Inc., Rochester, NY, USA) operating at 50W and $20 \mathrm{kHz}$ of power and frequency, respectively; the cell used for this purpose has been previously described and consists of a solenoid winding with honeycomb cell [4].

\section{Loading and release of the drug}

The two hydrogels PHE-Nip3 and AVA-5 were loaded with paroxetine and duloxetine according to the procedure previously described $[5,9]$. The loading of vortioxetine on hydrogels PHE-Nip3 and AVA-2 was conducted as follows. A weighed sample of dry hydrogel (PHE-Nip3, $100 \mathrm{mg}$; AVA-2, $50 \mathrm{mg}$ ) was swollen in distilled water containing a stoichiometric amount of sodium hydroxide. The fully swollen hydrogel was subsequently washed with distilled water and wiped with tissue paper. To the swollen hydrogel (EDS $=764$ for PHE-Nip3 and 352 for AVA-2) a solution $(20 \mathrm{~mL})$ of vortioxetine (101 mg for PHE-Nip3 and $174 \mathrm{mg}$ for AVA-2), previously filtered on cotton, was added. The addition of the drug solution caused a prompt collapse of the drug-loaded hydrogel which, after a few minutes, produced a finely dispersed solid. The solid residue was filtered and rapidly washed with distilled water three times (EDS $=1.2$ for PHE-Nip3 and 126.3 for AVA-2). After drying it in air and vacuum, the hydrogel provided an increase in weight consistent with the amount of loaded drug: PHENip3, 16.5 wt\%; AVA-2, 34.3 wt\%.

Pulsed release of the three drugs from the hydrogels considered was carried out at $25^{\circ} \mathrm{C}$ using three buffer solutions at different $\mathrm{pH}$ (pH 2.9, 4.6, and 7.4). For PHE-Nip3 hydrogel loaded with the drug, a quantity of dry sample $(4-10 \mathrm{mg}$ ) was weighed in a Strainer cell (pore size, $70 \mu \mathrm{m}$ ) and placed in $50 \mathrm{~mL}$ of the appropriate buffer at acidic $\mathrm{pH}$ (2.9 or 4.6) in a hermetically sealed plastic container and submitted to the AMF. A parallel control solution was prepared under the same conditions but without AMF. The amount of drug released at various set times was monitored spectrophotometrically by measuring the absorbance of the solution at $294 \mathrm{~nm}$ (for paroxetine), $290 \mathrm{~nm}$ (for duloxetine), and $227 \mathrm{~nm}$ (for vortioxetine). After three to four days of the observed slow release, the acid solution was rapidly replaced with $50 \mathrm{~mL}$ of PBS solution ( $\mathrm{pH} 7.40$ ); the Strainer cell containing the hydrogel was quickly washed in PBS and thoroughly wiped with tissue paper to measure the EDS value. The release of the drug was continued for a short period of one or two hours in PBS solution, measuring its absorbance value, and then resuming the initial acid $\mathrm{pH}$ solution to continue the release for further four days. Once again, the PBS solution was replaced for a short period of time and then placed in acid $\mathrm{pH}$ solution for further four days. The last substitution with the PBS solution continued the release of the drug for several days and until the drug completely discharged from the hydrogel that swelled to its maximum value under these experimental conditions. Obviously, at each step of replacing the PBS solution, weighing of the Strainer cell containing the hydrogel sample was carefully performed to determine the value of EDS. Similarly, but without the aid of the AMF, the pulsed 
release of drugs from the hydrogels AVA- 5 and AVA-2 was evaluated. The weighed sample quantities were 7-10 mg for AVA-5 and 5-6 mg for AVA-2. In all cases, at least two replicates for each sample were performed to evaluate drug release. The reproducibility of the results was always in good agreement.

\section{Acknowledgments}

This work was funded by the Department of Biotechnology, Chemistry and Pharmacy (University of Siena) with research support funds.

\section{Author contributions}

The project and the development of research, both experimental and theoretical, was cared for by Mario Casolaro. The manuscript was written together with Ilaria Casolaro who contributed for the psychiatric part and revised the entire paper in English.

\section{Conflicts of interest}

The authors declare no conflict of interest.

\section{References}

1. Casolaro M, Casolaro I (2013) Multiple stimuli-responsive hydrogels based on ?-aminoacid residues for drug delivery. In Smart materials for drug delivery; AlvarezLorenzo, C., Concheiro, A., Eds.; RSC Publishing: Cambridge, UK, 199-227.

2. Casolaro M, Casolaro I (2016) Stimuli-responsive hydrogels bearing ?-aminoacid residues: a potential platform for future therapies. J. Biom. Eng. Med. Dev 1: 1-18.

3. Casolaro M, Casolaro I (2012) Multiple stimuli-responsive hydrogels for metal-based drug therapy. Polymers 4: 964-985.

4. Casolaro M, Casolaro I, Bottari S, Del Bello B, Maellaro E, et al. (2014) Long-term doxorubicin release from multiple stimuli-responsive hydrogels based on ?-amino-acid residues. Eur J Pharm Biopharm 88: 424-433. [Crossref]

5. Casolaro M, Casolaro I (2015) Controlled release of antidepressant drugs by multiple stimuli- sensitive hydrogels based on ?-aminoacid residues. J Drug Deliv Sci Technol 30: 82-89.

6. Barbucci R1, Giani G, Fedi S, Bottari S, Casolaro M (2012) Biohydrogels with magnetic nanoparticles as crosslinker: Characteristics and potential use for controlled antitumor drug-delivery. Acta Biomater 8: 4244-4252. [Crossref]

7. Casolaro M (1995) Vinyl polymers containing L-valine and L-leucine residues: Thermodynamic behaviour of homopolymers and copolymers with $\mathrm{N}$-isopropylacrylamide. Macromolecules 28: 2351-2358.

8. Casolaro M, Paccagnini E, Mendichi R (2005) Stimuli-responsive polymers based on L-phenylalanine residues: Protonation thermodynamics of free polymers and crosslinked hydrogels. Macromolecules 38: 2460-2468.

9. Casolaro M, Casolaro I (2016) Polyelectrolyte Hydrogel Platforms for the Delivery of Antidepressant Drugs. Gels 2: 24.

10. Casolaro M, Cini R, del Bello B, Ferrali M, Maellaro E (2009) A cisplatin/hydrogel complex in cancer therapy. Biomacromolecules 10: 944-949. [Crossref]

11. Casolaro M, del Bello B, Maellaro E (2011) Hydrogel containing L-valine residue as a platform for cisplatin chemotherapy. Colloids Surf B Biointerfaces 88: 389-395. [Crossref]

12. Casolaro M, Casolaro I, Lamponi S (2012) Stimuli-responsive hydrogels for controlled pilocarpine ocular delivery. Eur J Pharm Biopharm. 80, 553-561. [Crossref]

13. Ohdo S (2010) Chrono-drug-delivery focused on biological clock: intra- and interindividual variability of molecular clock. Adv Drug Deliv Rev. 62: 857-858. [Crossref]
14. Khirwadkar P, Dashora K, Sisodiya D (2011) Chronopharmacotherapy: a night time therapy. Int J Pharm Sci Rev Res 9, 20-26.

15. Brazel CS, Peppas NA(1996) Pulsatile local delivery of thrombolytic and antithrombotic agents using poly(N-isopropylacrylamide-co-methacrylic acid) hydrogels. J Controll Rel 39: 57-64.

16. Peppas NA, Leobandung W (2004) Stimuli-sensitive hydrogels: ideal carriers for chronobiology and chronotherapy. J Biomater Sci Polym Ed 15: 125-144. [Crossref]

17. Kikuchi A, Okano T (2002) Pulsatile drug release control using hydrogels. Adv Drug Deliv Rev 54: 53-77. [Crossref]

18. Campbell S, Maitland D, Hoare T (2015) Enhanced pulsatile drug release from injectable magnetic hydrogels with embedded thermosensitive microgels. Macro Lett 4: 312-316.

19. Murray CJ, Lopez AD (1997) Alternative projections of mortality and disability by cause 1990-2020: Global burden of disease study. Lancet 349: 1498-1404. [Crossref]

20. World Health Organization: Mental disorders. Available online: www.who.int/ mediacentre/factsheets/fs396/en/.

21. Kupfer DJ, Frank E, Phillips ML (2012) Major depressive disorder: new clinical, neurobiological, and treatment perspectives. Lancet 379: 1045-1055. [Crossref]

22. Sugarman MA, Loree AM, Baltes BB, Grekin ER, Kirsch I (2014) The efficacy of paroxetine and placebo in treating anxiety and depression: a meta-analysis of change on the Hamilton Rating Scales. PLoS One 9, e106337. [Crossref]

23. Katona CL, Katona CP (2014) New generation multi-modal antidepressants: focus on vortioxetine for major depressive disorder. Neuropsychiatr Dis Treat. 10, 349-354. [Crossref]

24. Sonis WA (1992) Chronobiology of seasonal mood disorders. In Clinical guide to depression in children and adolescents; Shati, M., Ed.; Washington, DC, American Psychiatric Association, 1992, pp. 89-114.

25. Nemeroff CB (1994) The clinical pharmacology and use of paroxetine, a new selective serotonin reuptake inhibitor. Pharmacotherapy 14: 127-38.

26. Lu Z, Prouty MD, Guo Z, Golub VO, Cumar CSSR, Lvov YM (2005) Magnetic switch of permeability for polyelectrolyte microcapsules embedded with Co@Au nanoparticles. Langmuir 21: 2042-2050. [Crossref]

27. Hu SH, Liu TY, Huang HY, Liu DM, Chen SY (2008) Magnetic-sensitive silica nanospheres for controlled drug release. Langmuir 24: 239-244. [Crossref]

28. Liu J, Zhang Y, Wang C, Xu R, Chen Z, Gu N (2010) Magnetically sensitive alginate-templated polyelectrolyte multilayer microcapsules for controlled release of doxorubicin. J Phys Chem C 114: 7673-7679.

29. DrugBank: small molecule drugs. Available online: www.drugbank.ca/drugs (accessed on 30 march 2017).

30. Knadler MP, Lobo E, Chappell J, Bergstrom R (2011) Duloxetine: clinica pharmacokinetics and drug interactions. Clin Pharmacokinet 50: 281-94. [Crossref]

31. Raskin J, Pritchett YL, Wang F, D'Souza DN, Waninger AL, et al. (2005) A double-blind, randomized multicenter trial comparing duloxetine with placebo in the management of diabetic peripheral neuropathic pain. Pain Medicine 6: 346-356. [Crossref]

32. Baldwin DS, Hansen T, Florea I (2012) Vortioxetine (Lu AA21004) in the long-term open-label treatment of major depressive disorder. Curr Med Res Opin 28: 1717-1724. [Crossref]

33. Sanchez C, Asin KE, Artigas F (2015) Vortioxetine, a novel antidepressant with multimodal activity: review of preclinical and clinical data. Pharmacol Ther 145: 4357. [Crossref]

34. Casolaro M, Imanishi Y, Ito Y, Kono K (1989) An insulin-releasing system that is responsive to glucose. J Controll Rel 10: 195-203.

35. Casolaro M, Bottari S, Cappelli A, Mendichi R, Ito Y (2004) Vinyl polymers based on L- histidine residues . Part 1 . The thermodynamics of poly(ampholyte)s in the free and in the cross-linked gel form. Biomacromolecules 5: 1325-1332. [Crossref]

Copyright: $(02017$ Casolaro M. This is an open-access article distributed under the terms of the Creative Commons Attribution License, which permits unrestricted use, distribution, and reproduction in any medium, provided the original author and source are credited. 